\title{
Recent Developments in Self-Validating (SEVA) Sensors
}

\author{
The Author \\ Manus Henry is the Deputy Director of the Invensys University Technology Centre for Advanced Instrumentation at the \\ Department of Engineering Science, Oxford University, Oxford, UK. Email: manus.henry@eng.ox.ac.uk.
}

\section{Keywords}

sensor validation, fault detection, fieldbus.

\begin{abstract}
This paper provides a brief overview of recent developments in the field of self-validating (SEVA) sensors. The fundamentals of SEVA are described, using the example of a dissolved oxygen sensor. UK standardisation activities are then discussed along with moves towards commercialisation. Finally, some recent research results based on a coriolis mass flow meter are presented.
\end{abstract}

\section{Introduction}

Over the last twelve years, Oxford University and the Foxboro Company have developed the concept of the SelfValidating (SEVA) sensor (Henry and Clarke, 1993). Papers, talks and industrial trials have resulted in a consensus among industrial users and vendors that the SEVA concept would be valuable in industrial practice. Accordingly over the last year there have been a number of initiatives leading to a proposed British Standard for on-line measurement quality assessment based upon SEVA. It is hoped that this will subsequently be made available at the European (Cenelec) and World (IEC) forums. At the same time, Invensys (the parent company of Foxboro and other automation companies) has committed to releasing commercial products based upon SEVA within the next year. In addition, research at Oxford is continuing to develop new theory and demonstrations of SEVA.

\section{The Self-Validating (SEVA) Sensor}

The Sensor Validation Research Group at Oxford began in 1988 to examine the impact of digital technology on instruments, and to local fault detection in particular. It developed a theoretical model of how a 'self-validating' or SEVA instrument should behave (Henry and Clarke, 1993). This assumes the availability of internal computing power for self-diagnostics, and of digital communications to convey measurement and diagnostic data. A generic set of metrics are proposed for describing measurement quality (fig. 1). For each measurement, three parameters are generated:

- The Validated Measurement Value (VMV). This is the conventional measurement, but if a fault occurs, the VMV is a corrected best estimate of the true measurand value.

- The Validated Uncertainty (VU). This is the metrological uncertainty, or probably error of the VMV. For example, if the VMV is $4.31 \mathrm{l} / \mathrm{s}$, and the $\mathrm{VU}$ is $0.05 \mathrm{l} / \mathrm{s}$, then the sensor is claiming that the true measurement value lies between $4.26 \mathrm{l} / \mathrm{s}$ and $4.36 \mathrm{l} / \mathrm{s}$ with $95 \%$ confidence.

- The Measurement Value Status (MV Status). Given the requirement to provide a measurement, even with a serious fault, the MV Status indicates how the current measurement has been calculated. It takes one of a small set of values, of which the most important are:

C CLEAR: the measurement has been calculated normally.

- BLURRED: live raw data is still being obtained, but the VMV has been corrected for some fault condition.

口 DAZZLED: a temporary state ; it is known that current raw data is uncorrelated with the true process variable (e.g. the input is saturated), but it is not (yet) known whether the condition is permanent. The VMV is projected from past history, and the VU increases with time to reflect the reduced confidence in this projected VMV.

a BLIND: like DAZZLED except that there is evidence to suggest that the loss of raw data is permanent.

The VMV, VU and MV Status are generated for each measurement output from the sensor. For example, many industrial sensors measure process temperature as well as (say) flow or pressure. The validity of each is distinct, and each will be affected by a fault in a different way. However, for maintenance purposes, a single Device Status parameter is also provided, which indicates the level of maintenance action currently requested by the sensor (None, Low, High, Critical), alongside any device-specific detailed diagnostics.

The most important indicator of measurement quality is the on-line uncertainty of each measurement, the VU. It is calculated based upon all error sources affecting the on-line measurement, including:

- $\quad$ The transduction itself - the mapping from the true process measurand to the observed transducer signal;

- $\quad$ The components used to manufacture the instrument; 
- The characterization procedure at the end of the production line, and/or in-situ calibration procedures;

- The instantaneous operating point, and process noise;

- The effect of any faults, whether instrument- or process-induced, after compensation has been applied.

Thus the VU provides useful information about measurement quality whether or not a fault has occurred. By contrast, diagnostics are only provided in the (hopefully) rare occurrence of a fault, and describe only the nature of the fault and not the impact on the resulting measurement quality.

Figure 2 illustrates how the SEVA interface behaves when a fault does occur. Data has been generated from a prototype dissolved oxygen sensor (fig. 3) based upon an electrolytic cell (Clarke and Fraher, 1997). After one hour a fault begins in which fouling occurs on the membrane separating the electrolytic cell from the process. This is a common fault in, for example, waste water treatment plants. The fouling results in a reduction in the permeability of the membrane to oxygen molecules, and the raw dissolved oxygen measurement slowly drops over many hours. However, this selfvalidating sensor has two electrodes. One is used continuously for oxygen measurement, while the other is pulsed occasionally (typically every half hour) to detect fouling. Modelling of the time response of the test electrode can be used to detect fouling, and to calculate the drop in membrane permeability, and hence compensate the measurement. Patrick Fraher's thesis (Fraher, 1997) also provides an uncertainty analysis of the normal measurement and the correction for fouling. Thus, for the SEVA device, when the fault occurs the VMV is corrected, and the VU (as shown by the shaded interval surrounding the VMV) is increased to indicate the reduced quality of the corrected measurement. At the same time the MV status is switched to BLURRED, indicating that the measurement is being corrected for a fault. An independent measurement of the true dissolved oxygen level is also shown, to demonstrate that the corrected measurement provides a reasonable estimate of the true process value.

Consider now the added value provided by the SEVA metrics to the user of the measurement, for example a water treatment plant operator or an automated control system, with responsibility for maintaining a certain level of oxygen, say 7.5ppm (e.g. to avoid environmental damage).

In the absence of any fault detection, all that is seen is the raw measurement, which drops rapidly. With no other indication of the true oxygen level, then countermeasures may be taken, such as switching on air pumps, which are in reality unnecessary. Alternatively, if a sensor fault is suspected, then emergency (and hence expensive) maintenance action will be required to check and repair the sensor. Possibly both actions will be necessary until the fault state of the sensor is resolved.

Suppose now the sensor has diagnostics, and thus is able to indicate that a fault has occurred, for example by a devicespecific error code. In this case it is known that the sensor is the cause of the apparent drop in oxygen. However, the impact of this fault on the measurement cannot be assessed by the user, and so until the sensor is repaired there is no way of knowing the true dissolved oxygen level. Hence again, to continue process operation, immediate maintenance action will be required.

Consider now the response of the SEVA sensor. The operator is informed that a fault has occurred (the MV Status changes to BLURRED), but a corrected measurement is provided. Its increased uncertainty indicates the reduced confidence in the corrected measurement. Various responses are possible, depending upon whether the corrected measurement with its increased uncertainty is considered acceptable, as determined by local operating criteria. If not, then maintenance action must be carried out immediately. If however, the corrected measurement is deemed acceptable, then plant operation can continue and maintenance can occur when convenient, at a lower cost. Further action might include action to increase the reported dissolved oxygen level to say 8.5ppm or 9ppm to ensure that the true measurand does indeed stay above 7.5ppm, despite the increase in VU.

To summarize, SEVA maximizes the availability of the measurement by providing on-line correction for faults. It further provides an estimate of measurement quality in a standard, generic form, thus enabling operational and maintenance decisions to be taken based on application-specific criteria, without detailed knowledge of the sensor fault modes.

\section{Standardization and Commercialization}

Recent years have seen much activity in digital communications for industrial automation, usually termed Fieldbus. A range of fieldbus specifications are now available as IEC and Cenelec standards (Wood, 2000). The various fieldbusses have different properties, strengths and weaknesses. Guides are available advising which fieldbus to use in which application, for example (BSI, 2000). However, measurement quality is not addressed in most of the fieldbus standards.

In 1999 a survey was carried out by SIRA (SIRA, 1999), funded by the DTI. It explored current and future requirements for fieldbusses, intelligent measurements and diagnostics among users and suppliers in the UK automation industry. It discovered a consensus on the need for a common standard for describing sensor diagnostics and measurement quality, 
and that the SEVA concepts were a suitable starting point. The survey recommended that a standard be developed by the British Standards Institute (BSI).

This request was taken up by the BSI's AMT/7 (Fieldbus) committee which drafted a potential standard in the first half of 2000. At the time of writing this draft is being voted upon, and the results are expected in December 2000. The draft standard was tabled at a recent IEC Fieldbus committee meeting, and this may lead to a new working group being set up to develop a global standard for on-line measurement quality. Note that the SEVA concepts themselves are independent of any particular fieldbus protocol, and can thus be used with any fieldbus. However, it will be necessary to agree for each fieldbus type the particular convention used to transmit SEVA data.

Although aspects of the work have been ripe for commercial exploitation for some time, there have yet to be any major product releases based on SEVA. Two key reasons can be identified:

- The slow uptake of fieldbus, particularly in the USA;

- $\quad$ The desire of users for SEVA to be an open standard, and not tied to one specific vendor (i.e. Foxboro).

As discussed above, there have been recent improvements in both areas, and commercialization is now being undertaken. Currently Oxford is working only with Invensys companies (including Foxboro), but it is understood that several instrumentation and system vendors also wish to develop products conforming to the SEVA standard. Naturally it is not possible at this stage to give details of the commercialization plans. However, it can be disclosed that several instruments, including pressure, flow and positioner products, will be released with SEVA interfaces from several Invensys companies. In addition, a range of control system hardware and software products will be developed to provide support for SEVA instrumentation. This is of course essential if full use of SEVA functionality is to be possible, for example in control and maintenance activities. The first range of products are due for release in 2001.

\section{Recent research: SEVA Digital Coriolis}

A first generation SEVA prototype based on the coriolis mass flow meter was developed at Oxford in the early 90s. This consisted of the vibrating coriolis flowtube (in line with the process piping), and the conventional commercial electronic transmitter attached to a PC (Henry et al., 2000). The prototype demonstrated an ability to detect and correct for several fault modes, as well as the generation of SEVA metrics. However, the identification of the major limitations and fault modes of the instrument, and their impact on measurement quality, provided motivation for improvements in the basic design. This led on to the idea of an all-digital transmitter, which has been developed at Oxford. The primary intention was to replace all the analogue circuitry (other than essential front-end op-amps) with a small number of digital components, and to carry out virtually all functionality within software.

An all-digital design is attractive not only for research purposes, but also for commercial implementation. Digital (and specifically audio) technology is available for mainstream consumer markets such as mobile phones in large volumes at low cost. The commercial appeal is clear: a product with a low component count and high software content, based on rapidly evolving, competitive, mainstream technology. Examples of the performance improvements in the new digital transmitter include the following (see Henry et al., 2000 for more details):

- Repeatability. The repeatability of the digital transmitter is good. For example, two meters (driving $25 \mathrm{~mm}$ and $50 \mathrm{~mm}$ flowtubes) were placed in series in a flow rig, and their mass totals compared. Back-to-back trials, where both meters receive the same start and stop totalisation signals, are effective for assessing repeatability as several error sources (e.g. the repeatability and accuracy of the flow rig) are eliminated. The difference between the meter totals over 20 consecutive batches of $300 \mathrm{~kg}$ at $1 \mathrm{~kg} / \mathrm{s}$ exhibited a standard deviation of $0.005 \%$.

- Flowtube oscillation control. A new non-linear oscillation control algorithm has been developed (Clarke, 1998) which provides excellent stability of flowtube operation, and good response to changes in the setpoint for the amplitude of oscillation. Using this algorithm, it is possible to maintain flowtube oscillation down to $0.3 \%$ of the conventional amplitude, drawing only $0.1 \mathrm{~mA}$ per drive coil, demonstrating the potential for low power operation.

- Batching from empty. Many processes (e.g. food or petrochemical applications) could benefit from the high accuracy provided by coriolis technology in the metering of batches of material. Often it is not practical to ensure that the flowmeter remains full of fluid throughout the batch. However, large errors are induced in conventional coriolis meters when 'wet and empty'. The digital transmitter contains design features and algorithms to improve robustness in these conditions. For example, in a series of $550 \mathrm{~kg}$ batch experiments where the flowtube starts empty (Henry et al., 2000), the conventional transmitter generated large errors in the total mass, with a repeatability of only $87.7 \mathrm{~kg}$. By contrast the digital transmitter generates minimal error and a repeatability of $0.6 \mathrm{~kg}$. This suggests that batching to/from empty may soon be practical using coriolis mass flow metering.

Another important condition which presents difficulties for coriolis meters is two-phase (gas/liquid) flow, which can induce flowtube stalling and/or large measurement errors. A recent project at Oxford has explored detecting and 
correcting for two-phase flow (Liu et al., 2000). A neural-net model has been developed to predict the mass flow error from several internal parameters. Over a range of flow, void fraction and temperature values, mass flow errors are reduced from up to $25 \%$ to within $2 \%$.

Figure 4 illustrates how the SEVA device responds to the onset of two-phase flow. With single phase flow (up to $t=8 \mathrm{~s}$ ), the mass-flow measurement is CLEAR and it has a small uncertainty of about $0.2 \%$ of reading. Once two-phase flow begins, the correction algorithm is applied, the measurement is set to BLURRED, and the uncertainty increases substantially, reflecting the reduced accuracy of the corrected measurement. The user thus has the option of continuing operation with the reduced quality of the corrected mass-flow rate, switching to an alternative measurement, or shutting down the process. Where aeration occurs sporadically, the batch total uncertainty will be a function of both the duration and severity of the two-phase flow periods. It is hoped that as the understanding of the effects of two-phase flow improve, better correction techniques can be developed with correspondingly smaller uncertainty bounds.

The driving force behind the improvements in sensor functionality is the digital technology revolution, and the Oxford group has maintained an interest in the digital technology advances. For example, fieldbuses are only local area networks, and there is considerable interest in leveraging the enormous investment in Ethernet and Web technologies for field communications. Accordingly, the latest prototyping platform developed at Oxford includes a Web server embedded within each instrument. Developed under the Valcard II EPSRC grant, the platform is based on the PC104 standard for embedded applications. To an off-the-shelf processor board is added an FPGA-based coriolis card developed at Oxford (fig. 5). The application software runs under the VxWorks Real Time Operating System (RTOS). Figure 6 shows a typical Web screen generated by this latest generation coriolis prototype. Measurement, validity, configuration, and diagnostic information are available globally (subject to access permissions) via a standard Web browser. This has obvious benefits for both users and vendors, allowing instrument specialists to examine suspect instruments anywhere in the world. The web-serving coriolis meter may be seen on the Oxford Web site: http://seva.eng.ox.ac.uk.

The digital transmitter is the first example of a 'second-generation' SEVA instrument, in which the knowledge gained through validating the current commercial transmitter has led to a much more robust design. This illustrates SEVA as a philosophy promoting continuous measurement quality assessment and improvement, having impact on all aspects of the sensor lifecycle (e.g. design, manufacturing, characterisation, installation, calibration), and not merely on-line operation.

\section{Acknowledgements}

The author would like to acknowledge the hard work, support and enthusiasm of colleagues at Oxford and Invensys, along with the financial support of Invensys and the EPSRC.

\section{References}

BSI (2000). Guide to the Evaluation of Fieldbus Protocols, British Standards Institute, 389 Chiswick High Rd London W4 4AL .

Clarke, D.W. and Fraher, P.M. (1997). A self-validating Dissolved Oxygen Sensor. Control Engineering Practice, ...

Clarke, D.W. (1998). Non-linear control of the oscillation amplitude of a Coriolis mass-flow meter. European Journal of Control. Vol. 4, No. 3, pp196-207.

Clarke, D.W. (2000). Intelligent Instrumentation. Transactions of the Institute of Measurement and Control, Vol. 22, No. 1 , pp3-27.

Fraher, P.M. (1996). D.Phil. Thesis, Oxford University.

Henry, M. P. and D.W. Clarke (1993), The Self-Validating Sensor: Rationale, Definitions and Examples. Control Engineering Practice, 1 (4), pp.585-610.

Henry, M. P, Clarke, D. W., Archer, N., Bowles, J., Leahy, M. J., Liu, R. P., Vignos, J., Zhou, F.B. (2000). A selfvalidating digital coriolis mass-flow meter: an overview. Control Engineering Practice, Vol. 8, No. 5, pp487-506.

Liu, R.P., M.J. Fuent, M.P. Henry, M. D. Duta. (2000). A neural network to correct mass flow errors caused by twophase flow in a digital coriolis mass flowmeter. Accepted for publication in Flow Measurement and Instrumentation.

SIRA (1998). The impact of Fieldbus and sensor fusion on flow measurement. DTI NMS Programme for Flow Measurement, Project OR9A Technical Awareness Studies.

Wood, G.G. (2000). UK activities in measurement validation and data quality. IEE Computing and Control Journal, Special Issue on Intelligent and Self-Validating Instrumentation, October 2000. 

Figure 1. Measurement quality metrics generated by the self-validating (SEVA) sensor.

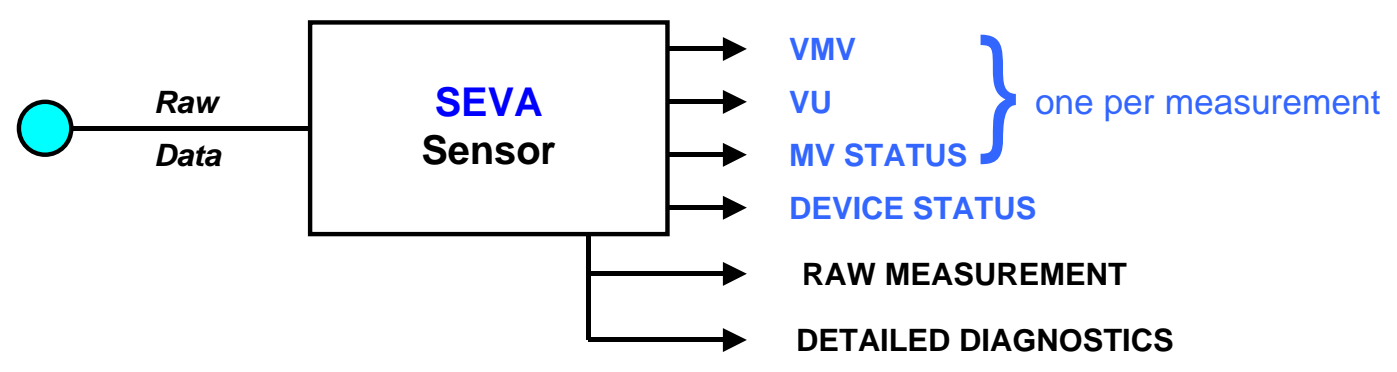


Figure 2. Response of SEVA Dissolved Oxygen sensor to fouling fault

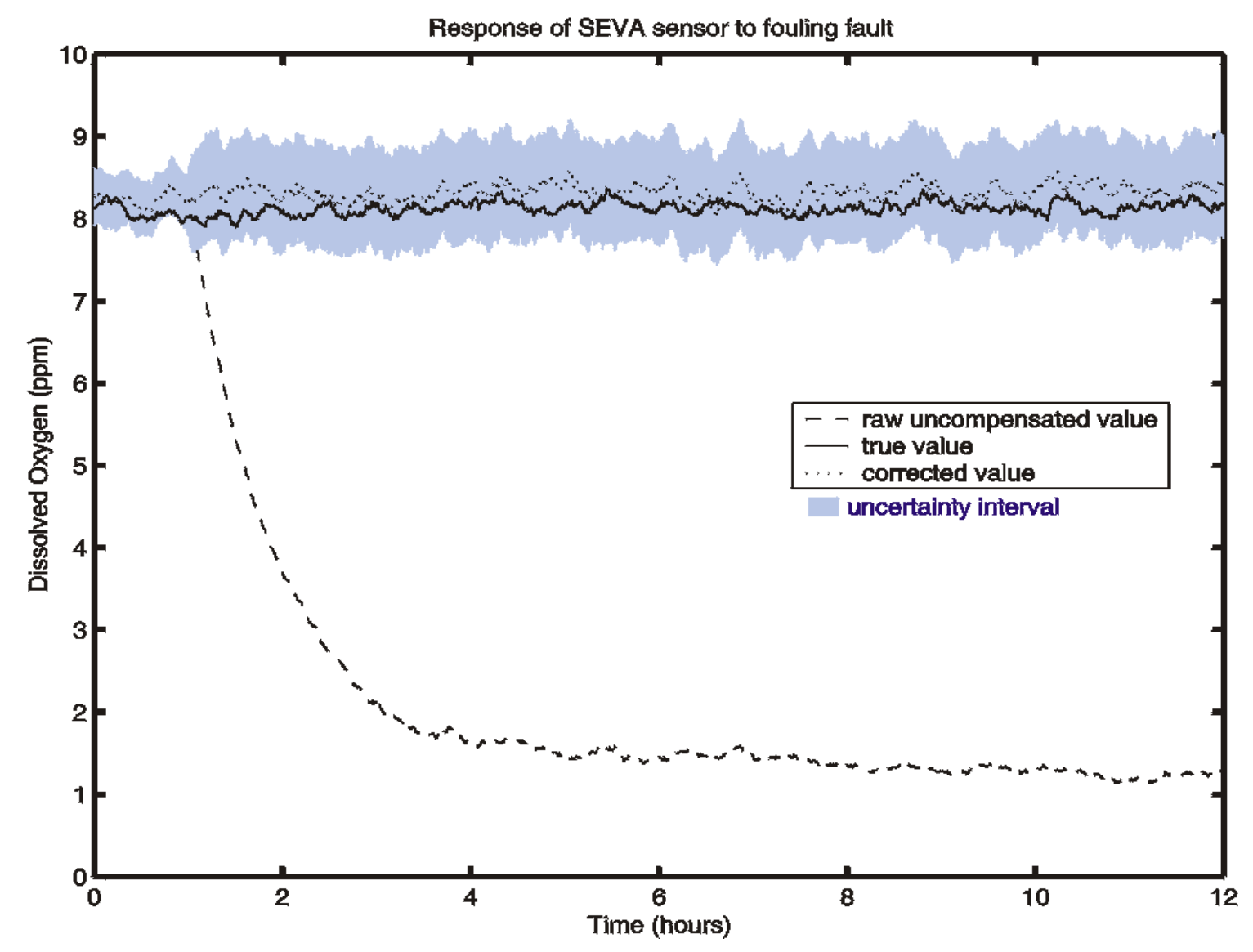


Figure 3. Oxford prototype SEVA dissolved oxygen sensor, with laptop Hand Held Terminal (HHT).

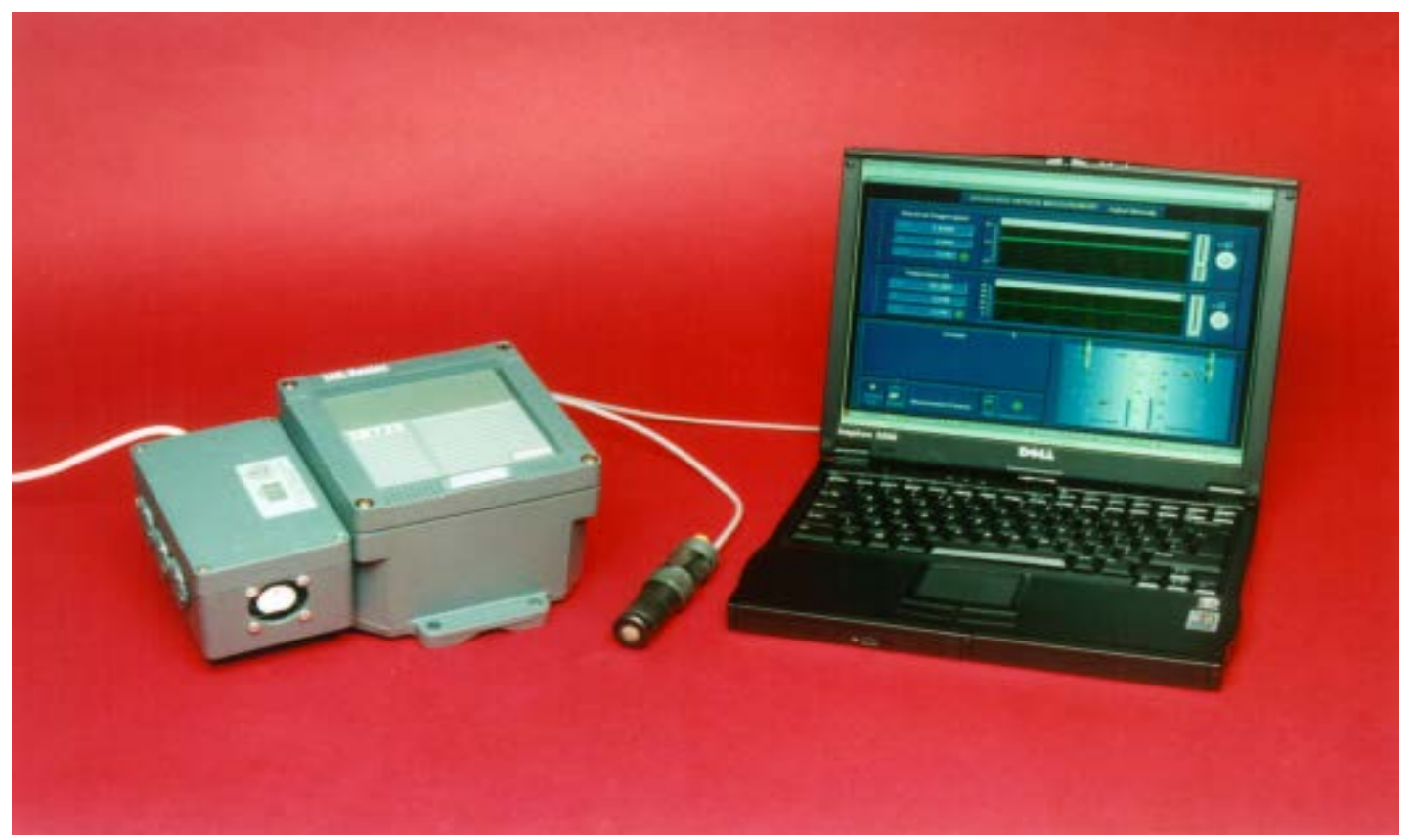


Figure 4. On-line response of self-validating digital transmitter to the onset of two-phase flow. Lower line: uncorrected massflow. Upper dark line: corrected massflow. Shaded region: uncertainty interval of corrected massflow. Dashed line: reading from master meter positioned prior to air injection point.

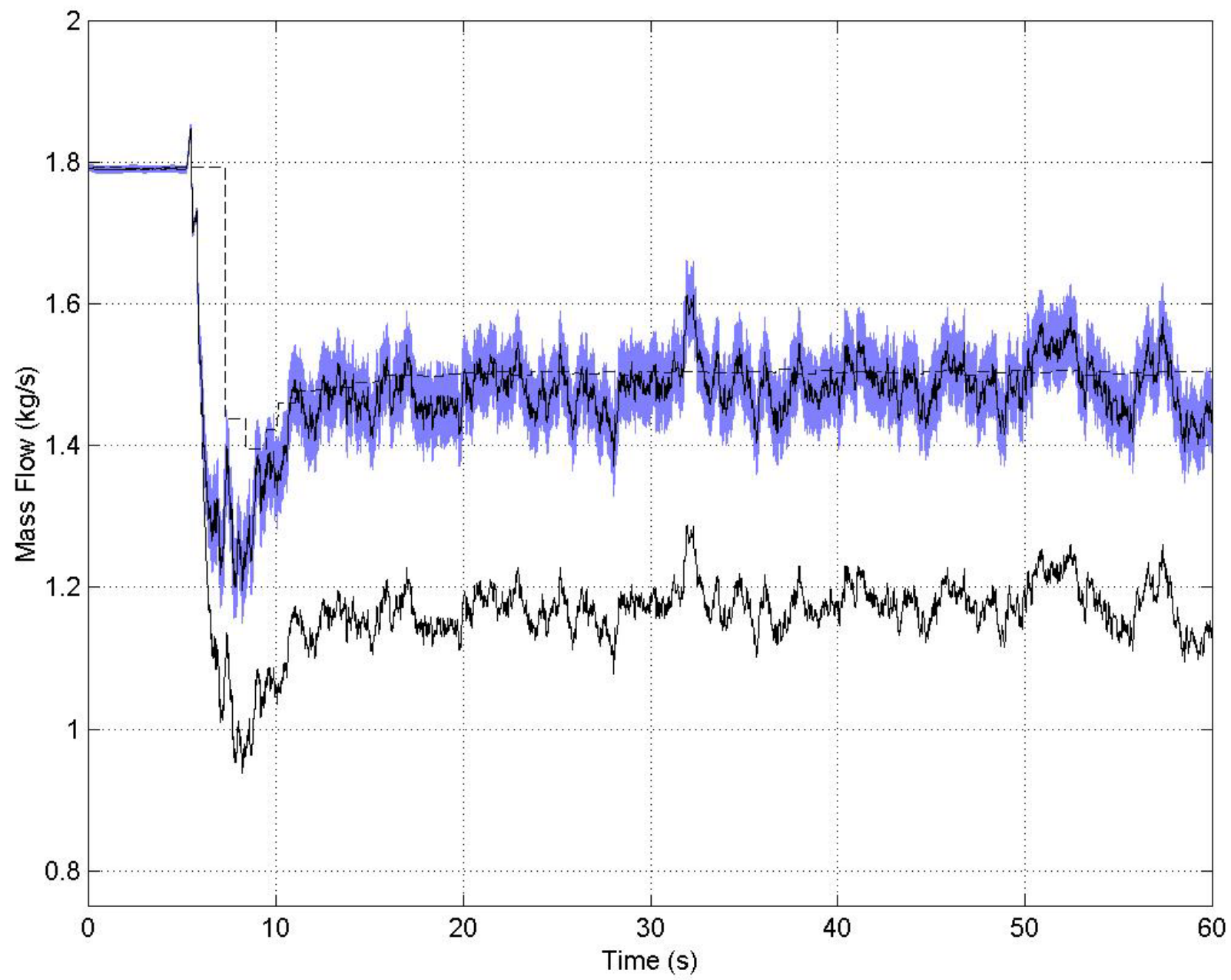


Figure 5. FPGA-based PC104 card for digital coriolis application.

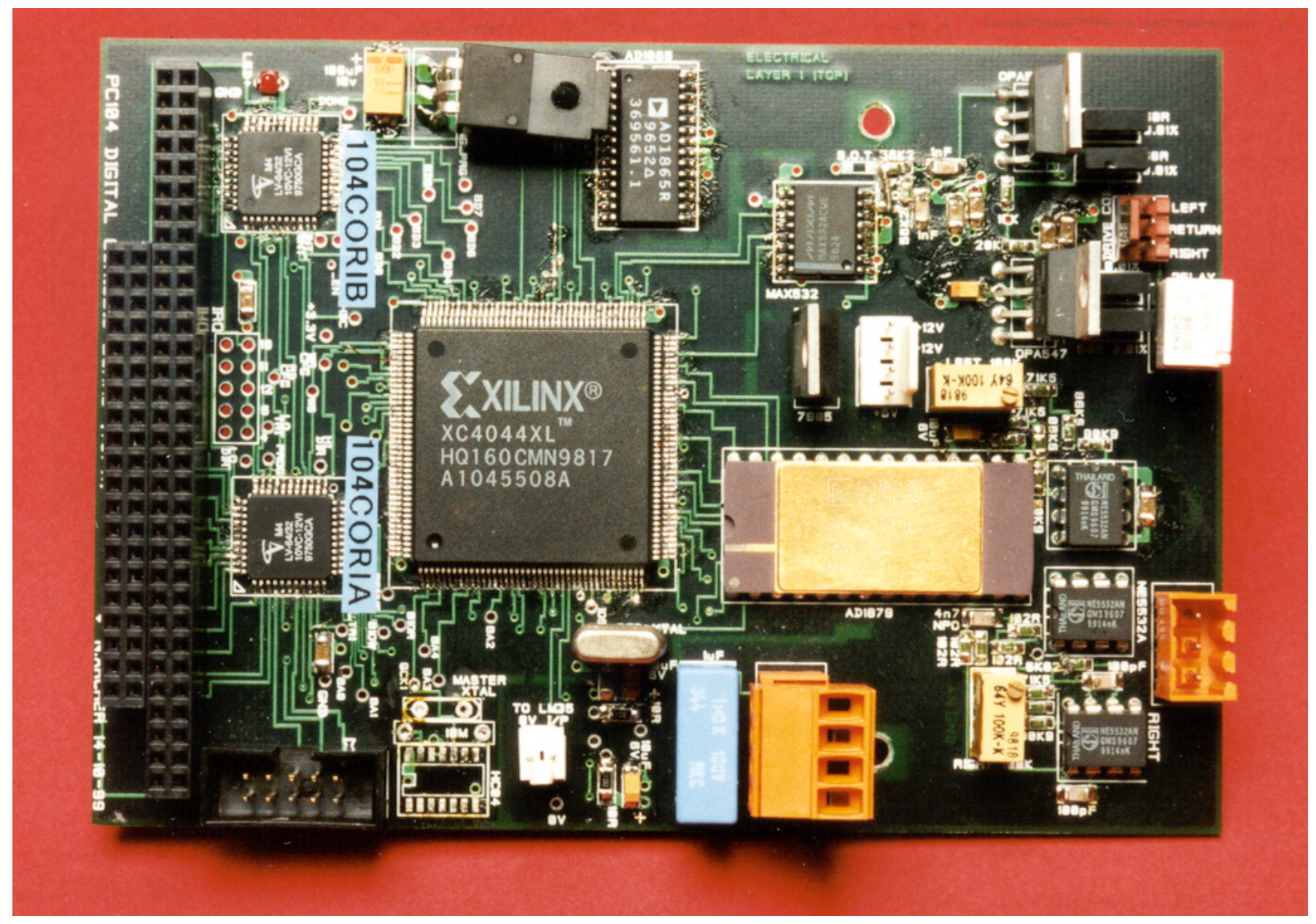


Figure 6. Web interface generated by digital coriolis prototype.

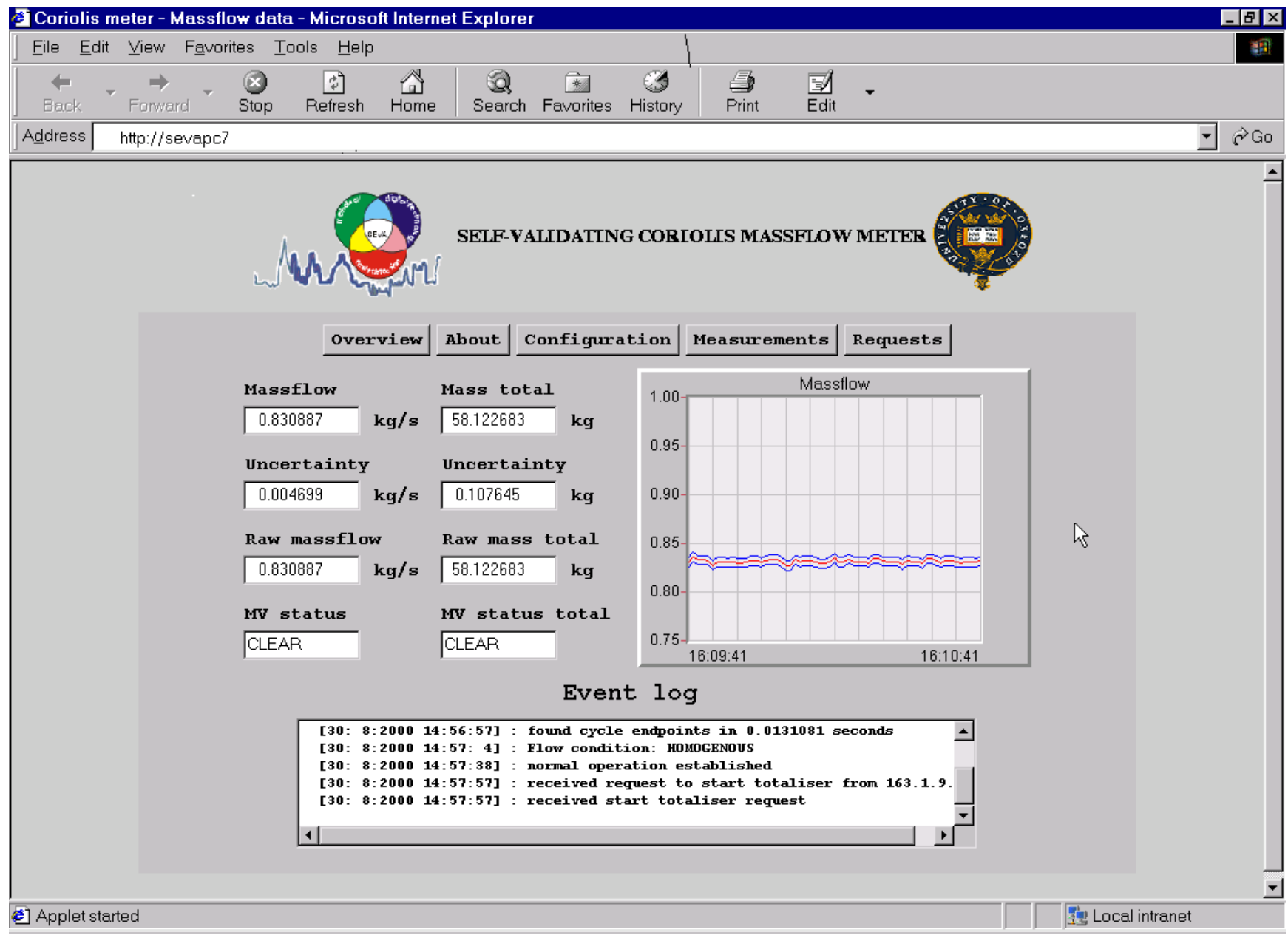

